\title{
Indoor-DSL: A Model Driven Approach to Modeling Indoor Representation Models for a Navigation Infrastructure
}

\author{
Evaldo da Silva Andrade \\ Federal University of Maranhao (UFMA) \\ Computer Science Postgraduate Program \\ Av. dos Portugueses, 1966 Bacanga \\ CEP 65080-805, Sao Luis MA Brazil
}

\author{
Samyr B. Vale \\ Federal University of Maranhao (UFMA) \\ Computer Science Postgraduate Program \\ Av. dos Portugueses, 1966 Bacanga \\ CEP 65080-805, Sao Luis MA Brazil
}

\begin{abstract}
Internal positioning and navigation tools provide important information about semantic aspects of buildings, however information about indoor maps construction is not usually available and designing tools used for modeling indoor environments are hard to use or expensive. In this article, a Domain Specific Language (DSL) for modeling indoor environments is presented, allowing to create internal representation models, independent of platform. This work aims to create indoor map models for infrastructure of Indoor Navigation System (INavigS), where all the domain concepts present in the tool are used to specify models. The principles of the Model Driven Approach (MDA) are applied to define a metamodel language. In addition, a graphical interface is provided for modeling indoor environment models used by INavigS, allowing to model internal indoor environments quickly and easily, keeping the focus on concerns related to the domain of navigation infrastructure.
\end{abstract}

\section{General Terms}

DSL, Indoor modeling environment, MDA

\section{Keywords}

Domain-Specific Language, Indoor representation model, Model-Driven Engineering, Modeling language, Indoor Navigation

\section{INTRODUCTION}

The world population has increasing in the last decades, reaching 7.7 billions of people in 2019 [24], and parallel to this growth, there is also an increase in the number of existing buildings, playing an important role in the economy of some nations [31]. Whereas about 80 to $90 \%$ of people spend their time inside buildings every day [21]. Thus increasing the need for structural spatial information and semantic information from these environments, in order to promote several kinds of applications focused on Indoor Positioning System (IPS), ranging from an autonomous vehicles on a factory to health care aid system into a hospital [4].
In regards about the formats of representation of an indoor environment, some approaches stand out into literature that can be used to provide internal space details of buildings, being Industrial Foundation Classe ${ }^{1}$ (IFC), CityGML Indoor Application Domain Extensions (ADE) [18] and IndoorGML ${ }^{2}$ Each one can be used to represent building elements in detail, as geometry and architecture, however only indoorGML exhibits a topological representation for indoor elements of build, which is suitable to navigation.

Traditionally, architectural software provides tools to draw floor plans through symbols, each one of elements as doors, floors, windows, all are represented by a Computer-Aided Design (CAD) symbol or polygons. However, there are no topological information of building, which is crucial for IPS applications that use topology-based navigation. Besides, translate CAD drawing into Geographic Information System (GIS) coordinates or build an indoor space representation model without the floor plan is a difficult and error prone task. There are some solutions to convert existing digitalized drawings $\mathrm{CAD}$ maps into navigation graph for indoor space [41], or indoorGML topological and semantic mapping from building plans data extraction [?]. This approach requires a lot of users interaction until reach the complete indoor representation model, in order to reduce the amount of interactions, in [20] is proposed a web based map design tool to make the user experience more pleasurable. Users provide a DFX file as input, and all data (e.g. polygons, doors, walls, and text) are extracted, resulting into a IndoorGML file.

In the literature, it is possible to find other alternatives for building indoor space representation model, using autonomous robots equipped with sensors which is proposed by [42] [34] or even mapping environments with laser scanners [40], and using the Simultaneous Localization and Mapping (SLAM) technique to obtain the indoor representation model. These approaches produce a detailed map, however it demands a high cost investment in equipment (e.g. laser sensor, cameras, robots). The construction of models to represent an internal environment that can be used in IPS becomes a challenging task for users who are unaware of CAD

\footnotetext{
$\overline{1_{\text {www.ifcwiki.org/ }}}$

${ }^{2}$ http://www.indoorgml.net/
} 
tools or even for the lack of an architectural drawing which can serve as a basis for elaborate a topological model.

Several tools have emerged in order to be used in IPS, the Indoor Navigation System (INavigS) is one of them. The INavigS it consists a software infrastructure used to provide position and route guidance to users $|25|$. Based on the Bluetooth Low Energy (BLE) beacons that are scattered around the environment. With a mobile device, the user can receive the signal from the beacon and estimate their relative position or receive assistance when traveling along a route. The infrastructure uses a topological representation of indoor model, where building elements are associated into Bluetooth beacons. All information are stored on relational database.

The current way to populate the database that contains the indoor representation model used by INavigS is made manually through Structured Query Language (SQL) statements. This task is assigned to the domain expert, being exhaustive and dismissive when the number of elements to be inserted into the model increases, in addition to making it difficult to trace relationships between elements on large models occasions.

The contribution of this paper is to provide a computer programming language with limited expressiveness and focused on a specific domain [11]. This Domain Specific Language (DSL) shall allow to create models of representation of the indoor space, aiming at the reduction of complexity and allowing users and domain experts to easily develop their own models through an intuitive, reduced and expressive graphic interface. Making the indoor environment modeling process simple and agile, instead time-consuming task of model with CAD tools, or even use expensive devices to execute this job. Firstly, it supports the development of indoor representation models used by INavigS infrastructure. The use of DSL will allow to create models of representation of the indoor space both by users and by domain experts that can be used by the INavigS navigation infrastructure. In addition to making it possible to increase productivity, by providing a graphical interface in which models can be created and manipulated, where the elements are represented solely for domain relevant aspects in a declarative way.

The rest of this paper is organized as follow. Section 2 describes some fundamental concepts related to software development in which the MDA based model is a central element. In Section 3 , the INavigS software infrastructure is presented, and also the overview of some applicability and indoor representation model used by them. Section 4 approaches the DSL aspects and notations. Section 5 demonstrates its use in some case studies, designing models for some proposed applications, and finally in Section 6, the paper conclusion is presented.

\section{FUNDAMENTAL CONCEPTS}

This section presents some works with different approaches to obtain the indoor space representation models. Therefore, it was sought to identify the main models of representation of the internal space and their respective formats [22] and ways to build a model that can contain indoor information [13]. In order to identify a way to fill the existing gap on INavigS navigation software infrastructure, since the information that constitutes the model used to represent the indoor space is cataloged and inserted individually in a database, becoming a difficult and exhausting task to be performed.
In recent years, the construction of a model of representation of the indoor space has attracted the attention of studies and commercial products. While some solutions make the model more expensive for using a large amount of equipment, others choose collaboration as a mechanism for obtaining data through the Volunteered Geographic Information (VGI) [39], in which people and sensors act together, being the main agents to provide data of indoor environment mapping. One of the main initiatives is the Open Street Maps (OSM) 3 project, which provides the possibility to edit indoor maps for free. All geometry is represented through nodes, ways, relations and tags and applied in indoor environment with indoor OSM extension. Although, indoorOSM can provide a mechanism for creating models that may represent the indoor environment, it still comes up against the large amount of interactions that need to occur to obtain such model, making the creation process difficult. In addition to the need for a floor plan to guide the process of creating a geographic model that can represent the indoor space using a floor plan editor like Potlatch [27] and JOSM [32].

Others initiatives start from the concept that the internal representation can be obtained from the floor plan, where the elements that make up the CAD drawing can have their characteristics extracted for the construction of the topological map [41], model mainly used in IPS navigation applications. In [1] a $\mathrm{C}++$ graphical application was developed having a CAD DXF file as input to compute nodes and edges that represent navigable spaces and interconnections between them, in this application all information is stored in a database. A similar approach was used in [35] to generate an internal model containing the geometric, topological and semantic information of buildings from existing data, using an input file in CAD DXF format, in which the file is interpreted to remove the elements. After this extraction step, the topologies and space geometry relationships are created, so that the semantic classification about this topology occurs, and results in an IndoorGML file. In [23] a pre-processing step was proposed to deal with errors regarding the representations of the existing walls in the CAD drawing, before of indoor spatial elements extraction step. After that, all topological information is obtained from the extracted elements.

Bearing in mind that the difficulty related to the extraction of characteristics of floor plans as a source of information to develop a model of representation of the indoor space resides in the large amount of interactions that must be performed by the user. In addition to the complexity associated with tools usage in the extraction process in the extraction process, or even on occasions when there is no floor plan with the information to elaborate the map. It becomes evident the need for alternatives that could reduce a complexity associated with the development of the representation model for indoor environments. This can be achieved by Model Driven Engineering (MDE) methodology in which software artifacts are generated conforms to model specification, models can also be designed independently of the target platform. The main principle in MDE are models, which is defined as partial or simplified representation of reality [2] allowing predictions or inferences to be made [19].

The use of MDE principles should improve quality, increasing traceability between artifacts, increasing productivity, maximizing compatibility between systems and facilitates the exploration of models to simulate, estimate, understand, communicate and generate code in an automated way [8]. To achieve such

$\overline{{ }^{3} \text { https://www.openstreetmap.org/about }}$ 
improvements, firstly increasing the level of abstraction, in which domain concepts are used directly in the elements specified by the language. Secondly, the high level of these abstractions is responsible for generating final software artifacts [15].

Models play a central role in MDE, a natural subsequent step in defining models and representing the models themselves as instances of some metamodel. Although in theory there is an infinite number of models. In practice the model proposed by the Object Management Group (OMG) ${ }^{\sqrt{4}}$ divides the modeling languages into a four-layer architecture and supported by the Meta Object Facility (MOF). This concept gave rise to a framework proposed by OMG for development of systems using MDE practices, called Model Driven Architecture (MDA) 5 where is provided a methodology to define and produce software based on model transformations. The above concepts allow to infer which a DSL of a system can be seen as a metamodel for a software which intends to be modeled. In addition, an environment must be defined so that users can specify their own models, through a well-defined language with form (syntax) and meaning (semantics) [29]. For [38] the main ingredients of DSL are:

Concrete Syntax: define notations that the user can specify models, that can be graphical, textual or both

Abstract Syntax: the data structure which keeps all relevant semantic.

Static Semantics: define a set of constrains and/or rules to which model to conform.

Execution Semantics: refers to the meaning of a program once it is executed

For [16] some advantages can be achieved by using DSL, among them the shorter time to market, faster customer feedback loops, lower development costs, allowing later changes, and new customer segments can be addressed more quickly.

\section{THE INAVIGS SOFTWARE INFRASTRUCTURE}

The INavigS is a context-aware software infrastructure supported by mobile devices that aims to provide services for people navigation in an indoor environment. The technology was developed into a research project of Laboratory of Intelligent Distributed Systems (LSDi) ${ }^{6}$ at the Federal University of Maranhao (UFMA). That architecture make uses of Mobile Hub (M-Hub) middleware that was developed by researchers from the Laboratory For Advanced Collaboration (LAC) 7 of the Pontifical Catholic University of Rio de Janeiro (PUC-Rio) [26]. The INavigS development idea came from the need for technologies that can allow a means of navigation in indoor environments, in which GPS does not work satisfactorily due to the fact that it was not created for indoor navigation support. The main services offered by the infrastructure are:

Geocoding: convert geographical positions (latitude and longitude) into semantic information (Room 202, Laboratory).

Route Estimate: obtaining positioning from the current position of the mobile device, and thus provide guidance on the path to be taken.

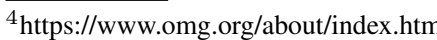

${ }^{5}$ https://www.omg.org/mda/specs.htm

${ }^{6} \mathrm{http}: / / \mathrm{www} .1$ sdi.ufma.br/

${ }^{7}$ http://www.lac.inf.puc-rio.br/
}

Providing information about the internal space: existence of elevators, ramps, stairs.

Route assistance: guidance information (turn left, turn right, go forward)

Its navigation hardware resources are composed in great majority of BLE beacons. A beacon can receive signals from different sources simultaneously and this information allows estimating the distance between these sources using the Received Signal Strength Indicator (RSSI), in other words, the greater the intensity of the received signal, the closest is the distance between sender and receiver. This is a proximity based location technique, in the literature it is possible to find similar approaches using other wireless communication technologies, like Radio Frequency Identification (RFID) [12], and Wireless Local Area Network (WLAN) [7], those technologies can be used individually or together in a complementary way, including different data sources, such as Bluetooth and Wi-Fi [5].

In INavigS case, Bluetooth beacons are used by the software infrastructure to provide navigation, each element of the building can receive one or more beacons. After established the position of Bluetooth devices by the environment, INavigS can provide navigation, route calculation and geocoding applied in different domains, such as navigation in a hospital [6], system to locate products in stores [17] or even guide tourists through a museum or city [33] its application in different scenarios is shown in Figure 1.

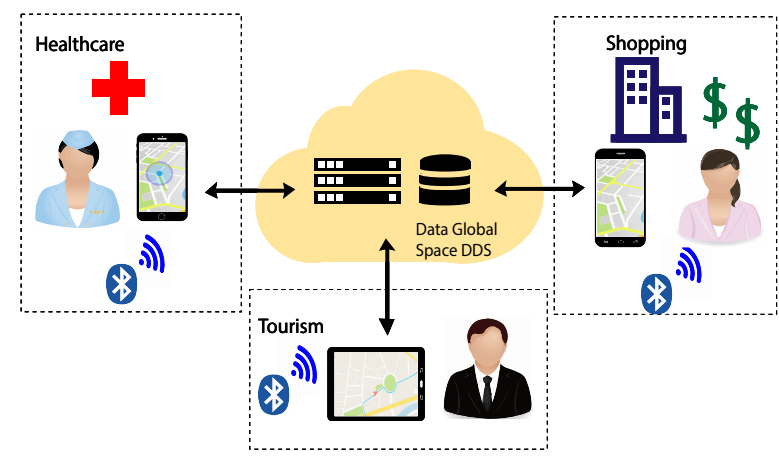

Fig. 1. Different domains where INavigS can be used.

Regarding the representation of the spatial model, it occurs through a hierarchical symbolic spatial model, as described in Figure 2 The space is divided into three layers: Building, where the information describing the building are found (Building Name, Address). BuildingElements to describe the entrances, exits, hall, bathrooms, corridors and stairs, all possible elements which compose a building. At the level of Beacons and Links, the existence of beacons and the connections between them are demonstrated. Each beacon has a Unique Universal Identifier (UUID), which can be used for identification, besides latitude and longitude, whereas the Links are described by a value which represented the distance between beacons, measured in meters.

The creation of the spatial model is illustrated by Figure 3 . Where $P 0, P 1$ and $P 3$ are representing beacons that have been positioned in the environment. To perform the calculation of the coordinates of all the mapped points, some steps are necessary. Firstly, the geographical coordinates of the initial position $(P 0)$ are obtained, as well as their semantic representation (e.g. classroom 


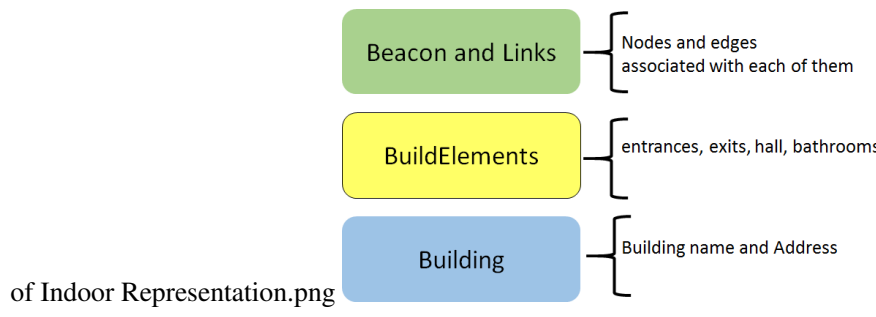

Fig. 2. Hierarchical Model of Indoor Space used by INavigS.

04, computer coordination room). Secondly, the values of the azimuths represented by the angles (alpha and beta) are obtained. Afterwards, the value of the distances $d 1$ and $d 2$ for the beacons positioned in the environment ( $P 1$ and $P 2$ ).

of spacial model.png

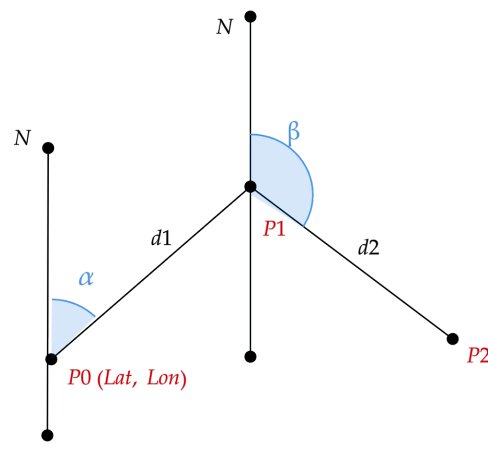

Fig. 3. Elaboration of spatial model used by INavigS software infrastructure.

With this approach of building the model of representation of indoor space, the adoption of a CAD model becomes unnecessary, as well as facilitating the implementation of a navigation infrastructure on occasions when there is no floor plan available. To calculating the route to obtain the shortest path is used the Dijkstra algorithm on the graph,since the topological information that represents the indoor space is organized in a graph data structure. where the nodes are Bluetooth beacons and the edges are represented by the distance between the beacons in meters.

Despite to the fact that INavigS solves the problems of calculating and obtaining the route, some gaps remain making it difficult to use, such as in case of building indoor space representation model which must contain the architectural elements of the building, sensors and links, all objects that compound the model by the referred software infrastructure. It demands extra effort by the domain specialist, with the responsibility of manually entering all the information, one by one, directly into the database manually. Thus, it becomes evident that the need for tools that can speed up the process of developing environmental models at the same time, can provide means for users without any knowledge of the domain to model their own indoor representation models. The development of a tool focused on resolution of this particular problem of INavigS for indoor representation model construction is described into following sections.

\section{THE INDOOR-DSL MODELING LANGUAGE}

The modeling language was conceived through Obeo Designer ${ }^{8}$ a tool in which it is possible to specify the metamodel for using models on model driven process. The framework uses models to describe graphic elements and tools to manipulate those elements. Its development comprises the steps described in [37], as being the following:

Analysis: once the problem is identified, in the case of INavigS, the absence of a tool that allows the creation of models to represent indoor space at a high level of abstraction. The analysis phase is responsible for conducting a search of all relevant information to the domain. This phase can be performed in an ad-hoc manner, in which the domain specialist performs the analysis task because he is intimately familiar with the characteristics of the domain [14]. Crucial information for designing a DSL in which the applications inherent to the domain can be modeled.

Implementation: This phase consists on the development of the tools that will be responsible for the generation of code and transformation between models.

Use: modeling domain applications using DSL.

The conversion of applications developed using DSL can be achieved through the Acceled 9 tool, allowing to perform Model-To-Text (M2T) transformations, where templates can be used to produce any type of source code (e.g. JAVA, C,++ C) from EMF data format.

\subsection{Abstract Syntax}

The abstract syntax of a DSL was developed by using the Eclipse Modeling Framework (EMF ${ }^{10}$ metamodel language used to produce the ECore Indoor-DSL metamodel. The Ecore was used to define classes, relations, attributes and data types. All the elements are composed of concepts and relationships raised from domain, as depicted in Figure 4



Fig. 4. IndoorDSL Metamode

\footnotetext{
${ }^{8}$ https://www.obeodesigner.com

${ }^{9}$ https://www.eclipse.org/acceleo/overview.html

${ }^{10}$ https://www.eclipse.org/modeling/emf/
} 
The initial element of the metamodel is the "ObjectModel", from it, the "Address" and Link elements can be specified. After defining the respective values, the element Build can be created from the element ". To instantiate an object of type "BuildElement", there must be a "Build" element created. After creating "Address", "Build" and "BuildElement", a "Beacons" instance can be made, in which each beacon is assigned to the respective "BuildElement", with respect to the "Link" object once created, "Beacons" can be bound with one or more links.

\subsection{Concrete Syntax}

A graphic representation was developed containing the representation of the concepts defined in the abstract syntax. For its elaboration, the Sirius ${ }^{11}$ environment provided by EMF was used, significantly reducing the effort to develop the user interface, by providing the possibility to use the model as reference to graphical notation. The view from model offered by Sirius is called the viewpoint specification, and it is organized in a tree of objects allowing to determine the behaviour in relation to the structural aspects of the metamodel. In Indoor-DSL Modeling Language case, the viewpoint is called "InavigsDSL", as described on Figure 5

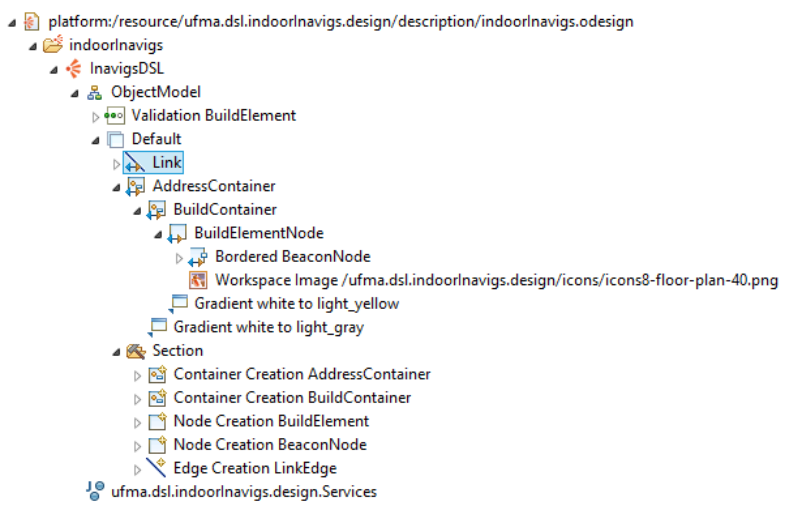

Fig. 5. Sirius viewpoint specification of IndoorDSL.

All elements described in metamodel must be included on Sirius "viewpoint specification", its are "Link", "AddressContainer", "BuildContainer", "BuildElementNode" and "BorderedBeaconNode". Some attributes used to identify the objects as "idAddress", "idBuild", "idBuildElement", "idBeacon" and "idLink" (See metamodel 4) receive some expressions written in Acceleo Query Language (AQL) to ensure that there is no repetition in the identification values, making sure that the last valid identification value is always assigned. To obtain the value, the expression used is "aql:self.eResource().getContents().eAllContents()"+ "EntityFilter", where the "EntityFilter" corresponds to the entity filter represented in the model, the following filters are applied to define the identifiers for "idAddress" (See filter 1), "idBuild" (See filter 2), "idBuildElement" (See filter 3), "idBeacon" (See filter 4) and “idLink" (See filter 5).

$$
->\operatorname{filter}(\text { Address })->\operatorname{size}()
$$

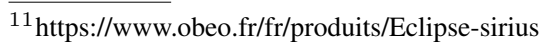

$$
\begin{gathered}
->\text { filter }(\text { Build })->\operatorname{size}() \\
->\text { filter }(\text { BuildElement })->\operatorname{size}() \\
->\operatorname{filter}(\text { Beacon })->\operatorname{size}() \\
->\operatorname{filter}(\text { Link })->\operatorname{size}()
\end{gathered}
$$

Objects inserted into a Sirius container receive the value of the container identification in which they are inserted. For example, the object BuildElement is inserted in the container Build, the idBuild attribute contained in the BuildElement object receives the value of the container in which it was inserted. AQL expressions related to Build (See expression 6, BuildElement (See expression 7), Beacon (See expression 8) respectively are:

$$
\begin{gathered}
a q l: \text { self.eContainer(Address).idAddress } \\
a q l: \text { self.eContainer(Build).IdBuild }
\end{gathered}
$$

$$
\text { aql : self.eContainer(BuildElement).IdBuildElement }
$$

In the case of Link, obtaining the values corresponding to the identifications of the interconnected beacons values for idBeacon1 (See expression 9) and idBeacon2 (See expression 10) are achieved by the expressions:

$$
\begin{gathered}
a q l \text { : self.from.idBeacon } \\
\text { aql : self.to.idBeacon }
\end{gathered}
$$

Based on the elements contained in the abstract syntax, the graphic representation of the main objects of IndoorDSL is obtained through the mapping between the elements of the metamodel and its respective graphic representation. All elements used to design a graphical notation are describe in the Table 1

Table 1. Graphical Notation Elements

\begin{tabular}{ll}
\hline Abstract Syntax/Object & Concrete Syntax/Icon \\
\hline Address & \\
Build & \\
BuildElement
\end{tabular}

\subsection{The Indoor-DSL Ovierview}

The Sirius EMF plug-in allows to create an entire graphical environment in a model driven development way. All graphical components specified are in accordance with domain under study. The designing process is carried out through the graphical interface illustrated in Figure 6 It consists on three different areas. 
1- Design area: in this area, users can create their own models freely.

2- Object Properties area: in this area there are all editable class attributes, a form with multiple entries is displayed when a particular object is selected.

3- The palette area: Pallet contains all the elements represented by the concrete syntax; they are used to create instances of the model; each element is associated with the elements presented in the metamodel.

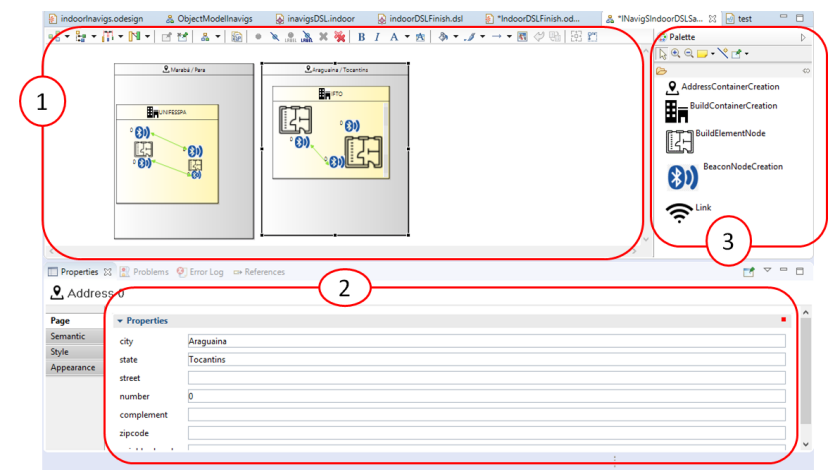

Fig. 6. The design interface screen of IndoorDSL.

\section{CASE STUDY}

To illustrate the use of Indoor-DSL, some use cases about indoor environment modeling are presented in different spaces and contexts.

\subsection{Modeling the Indoor Representation Model of Emergency Evacuation Response}

The IPS applications need topological information from the building to provide navigation assistance. In a disaster case, e.g. they can decrease the time spent looking for a specific exit [10]. A hospital wants to create a model to represent its architectural elements to guide patients and visitors inside the building in case of emergency. With 18 rooms, 2 halls, 1 entrance and 2 exits, each room has a beacon associated with it. Figure 7 depicts the possible scenario modeling by using Indoor-DSL.

\subsection{Modeling for a Public Building}

A public building needs a topological model of its environment to provide routes information for people with motor disabilities [30], with a total of 20 rooms, 1 staircase, 1 ramp, 4 corridors spread over 3 floors. Bearing in mind that each building element has at least one beacon to identify the element. The Figure 8 illustrates the proposed model for this scenario.

\subsection{Experiments and Results}

In addiction, some experiments are conducted to test Indoor-DSL modeling language, where users can create indoor model representations used by INavigS software infrastructure. A usability test has been performed in order to estimate the visual aspect of the domain language, in which it is performed empirically and non-empirically. Similar approach can be found in [9]. Since

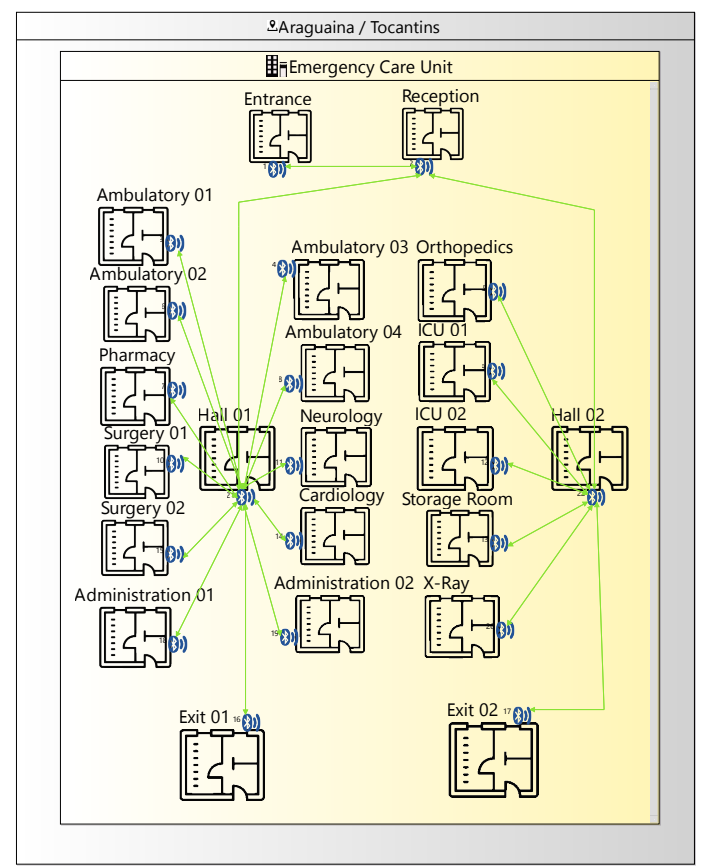

Fig. 7. Scenario of emergency evacuation response case modeled through IndoorDSL modeling language.

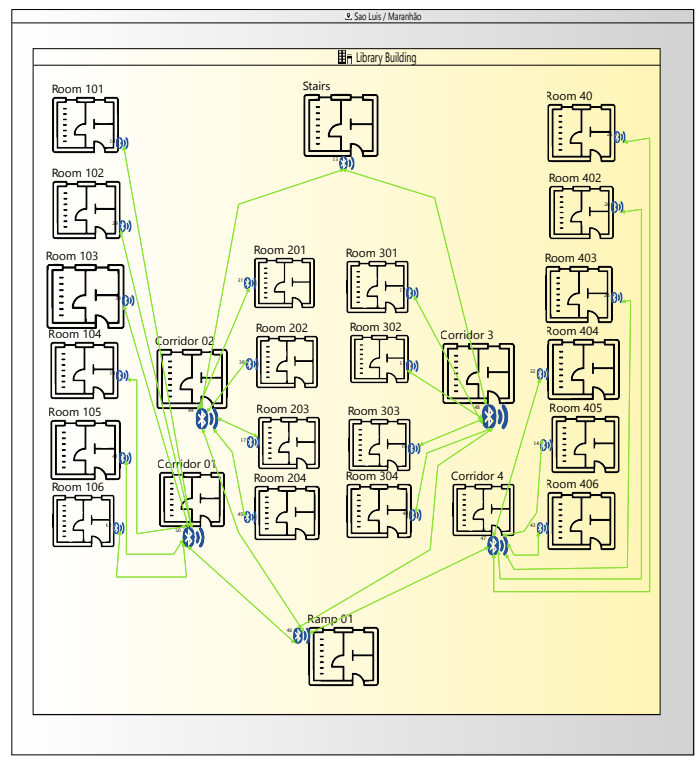

Fig. 8. Scenario of public building routes for people with motor disabilities modeled through IndoorDSL modeling language.

four or five users are responsible for discovering $80 \%$ of problems [36], assuming 8 participants of this phase, separated into 2 groups as follows:

-6 people with low or none knowledge of DSL, MDE and indoor representation model.

-2 people with regular previous experience into DSL, MDE and indoor representation model. 
A simple task was assigned to each participant in which the representation of the indoor space should be modeled, even simplistic, the model includes aspects that can be found in a real indoor environment, the System Usability Score (SUS) was taken into account as a measure [3]. The SUS was computed as [28], where the value obtained into evaluation was $\mathbf{8 6 . 8 7}$, considered as an excellent value in according to the SUS scale demonstrated in the Figure 9

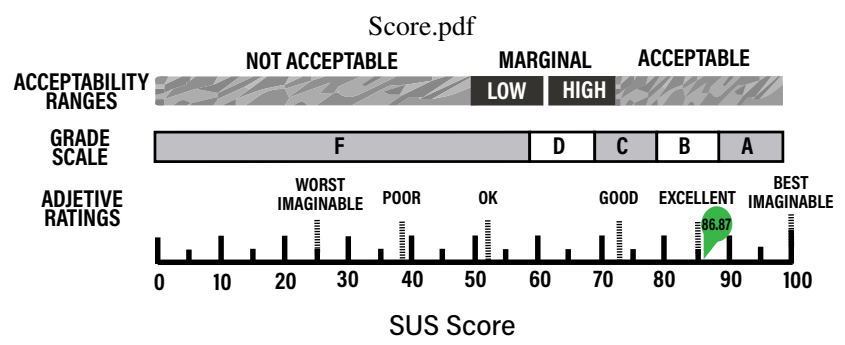

Fig. 9. SUS score scale with result obtained of IndoorDSL modeling Language.

For a better understanding of the evaluating results of the Indoor-DSL modeling language use, a histogram illustrated in Figure 10 shows the distribution of the percentages obtained with user feedback. As can be seen, most users rate the Indoor modeling language as excellent, even without having prior knowledge about the internal representation model.

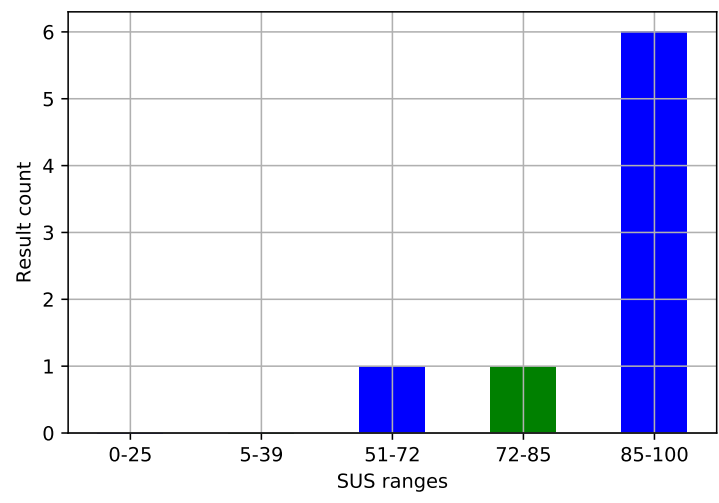

Fig. 10. IndoorDSL usage results distributed by SUS score ranges

\section{CONCLUSION AND FUTURE WORKS}

This article presents a new approach for development of indoor space representation model based on the model-driven development, providing an easy mechanism for creating models using DSL tailored to domain specifications. Thus allowing to create models quickly, which can be used for IPS infrastructures through a graphic language, the use was demonstrated on INavigS indoor representation model. Both experts and users without any knowledge of the technologies and tools for the elaboration of a model of representation of the indoor space can use this tool for developing their own models at a high level of abstraction.
Some features which could improve IndoorDSL modeling language have been noted, and left as suggestions to future Works, as transformation for different indoor representaiton models found in the literature, as well as the possibility of create new metamodels intended for different IPS systems and provide transformations between them.

\section{REFERENCES}

[1] Luciano Caraciolo Albuquerque. 2d vector map and database design for indoor assisted navigation. Master's thesis, CUNY City College, apr 2017.

[2] Marco Brambilla, Jordi Cabot, and Manuel Wimmer. Model-Driven Software Engineering in Practice, volume 1. 2012.

[3] John Brooke. Sus: a quick and dirty'usability. Usability evaluation in industry, page 189, 1996.

[4] Luca Calderoni, Matteo Ferrara, Annalisa Franco, and Dario Maio. Indoor localization in a hospital environment using random forest classifiers. Expert Systems with Applications, 42(1):125-134, January 2015.

[5] Hilário José Silveira Castro, Ivanovitch Medeiros Dantas da Silva, and Silvio Costa Sampaio. Uma metodologia de localização indoor para smartphones em ambientes de cidades inteligentes. In Anais do I Workshop Brasileiro de Cidades Inteligentes. SBC, 2018.

[6] Nidal Chalhoub and Gabriella Simon-Nagy. Indoor navigation based on linked data at honvéd hospital, budapest. In 2018 IEEE 12th International Symposium on Applied Computational Intelligence and Informatics (SACI). IEEE, May 2018.

[7] Ionut-Catalin Draghici, Andrei Vasilateanu, Nicolae Goga, Bujor Pavaloiu, Laurentiu Guta, Monica N Mihailescu, and Costin-Anton Boiangiu. Indoor positioning system for location based healthcare using trilateration with corrections. In 2017 International Conference on Engineering, Technology and Innovation (ICE/ITMC), pages 169-172. IEEE, 2017.

[8] Atli F. Einarsson, Patrekur Patreksson, Mohammad Hamdaqa, and Abdelwahab Hamou-Lhadj. SmartHomeML: Towards a domain-specific modeling language for creating smart home applications. In 2017 IEEE International Congress on Internet of Things (ICIOT). IEEE, June 2017.

[9] Teo Eterovic, Enio Kaljic, Dzenana Donko, Adnan Salihbegovic, and Samir Ribic. An internet of things visual domain specific modeling language based on UML. In 2015 XXV International Conference on Information, Communication and Automation Technologies (ICAT). IEEE, October 2015

[10] F. Fadli, N. Kutty, Z. Wang, S. Zlatanova, L. Mahdjoubi, P. Boguslawski, and V. Zverovich. Extending indoor open street mapping environments to navigable 3D citygml building models: Emergency response assessment. International Archives of the Photogrammetry, Remote Sensing and Spatial Information Sciences - ISPRS Archives, 42(4):241-247, 2018.

[11] Martin Fowler. Domain Specific Languages. Addison-Wesley Professional, 1st edition, 2010.

[12] Chung-Hao Huang, Lun-Hui Lee, Chian C Ho, Lang-Long $\mathrm{Wu}$, and $\mathrm{Zu}-\mathrm{Hao}$ Lai. Real-time rfid indoor positioning system based on kalman-filter drift removal and 
heron-bilateration location estimation. IEEE Transactions on Instrumentation and Measurement, 64(3):728-739, 2014.

[13] Watthanasak Jeamwatthanachai, Mike Wald, and Gary Wills. Map data representation for indoor navigation a design framework towards a construction of indoor map. In 2016 International Conference on Information Society (i-Society). IEEE, October 2016.

[14] Abdelilah Kahlaoui and Alain Abran. Demystifying domain specific languages. In Computational Linguistics: Concepts, Methodologies, Tools, and Applications, pages 228-250. IGI Global.

[15] Steven Kelly and Juha Pekka Tolvanen. Domain-Specific Modeling: Enabling Full Code Generation. 2007.

[16] Steven Kelly and Juha-Pekka Tolvanen. Domain-specific modeling: enabling full code generation. John Wiley \& Sons, 2008.

[17] Ismahne Hadj Khalifa, Abdelkader El Kamel, and Bernard Barfety. Real time indoor intelligent navigation system inside hypermarkets. IFAC Proceedings Volumes, 43(8):461 - 466, 2010. 12th IFAC Symposium on Large Scale Systems: Theory and Applications.

[18] Yun Ji Kim, Hye Young Kang, and Jiyeong Lee. Development of indoor spatial data model using CityGML ADE. International Archives of the Photogrammetry, Remote Sensing and Spatial Information Sciences - ISPRS Archives, XL-2/W2(November):41-45, 2013.

[19] Thomas Kühne. Matters of (meta-) modeling. Software \& Systems Modeling, 5(4):369-385, July 2006.

[20] Juhani Lavonen. Developing a web application for indoor map data management. Master's thesis, Metropolia Ammattikorkeakoulu, may 2018.

[21] Ki-Joune $\mathrm{Li}$ and Jiyeong Lee. Indoor spatial awareness initiative and standard for indoor spatial data. In Proceedings of IROS 2010 Workshop on Standardization for Service Robot, volume 18, 2010.

[22] Ki-Joune Li, Sisi Zlatanova, J. Torres-Sospedra, A. Perez-Navarro, Christos Laoudias, and Adriano Moreira. Survey on indoor map standards and formats. In 2019 International Conference on Indoor Positioning and Indoor Navigation (IPIN). IEEE, September 2019.

[23] Zhixing Lin, Chundi Xiu, Wei Yang, and Dongkai Yang. A graph-based topological maps generation method for indoor localization. In 2018 Ubiquitous Positioning, Indoor Navigation and Location-Based Services (UPINLBS), pages 1-8. IEEE, March 2018.

[24] Hannah Ritchie Max Roser and Esteban Ortiz-Ospina. World population growth. Our World in Data, 2013. https://ourworldindata.org/world-population-growth.

[25] Allinger Lima Medeiros. Inavigs: uma infraestrutura de software ciente de contexto para navegação indoor. Master degree thesis, Universidade Federal do Maranhão, 2018.

[26] L. C Muniz. M-hub. Available at www.lsdi.ufma.br/ projetos/cddl/doku.php (accessed 2020.06.13).

[27] OpenStreetMap. Potlatch 2. Available at https: //wiki.openstreetmap.org/wiki/Potlatch_2 (accessed 2020.05.22).

[28] Lucio Padrini-Andrade, Rita de Cássia Xavier Balda, Kelsy Catherina Nema Areco, Paulo Bandiera-Paiva, Marynéa do Vale Nunes, Sérgio Tadeu Martins Marba, Werther Brunow de Carvalho, Ligia Maria Suppo de Souza Rugolo,
João Henrique Carvalho de Almeida, Renato Soibelmann Procianoy, et al. Avaliação da usabilidade de um sistema de informação em saúde neonatal segundo a percepção do usuário. Revista Paulista de Pediatria, 37(1):90-96, 2019.

[29] Pimenta Paulo. Application of Model-driven engineering to multi-agent systems: a language to model behaviors of reactive agents. Thse de doctorat : Informatique : Universit de montpellier, Montpellier : Universit de Montpellier, 2017.

[30] Celso Pereira, António Sousa, and Vítor Filipe. Open-Source Indoor Navigation System Adapted to Users with Motor Disabilities. Procedia Computer Science, 67(Dsai):38-47, 2015.

[31] Chris Rhodes. Construction industry: statistics and policy. House of Commons Library, (01432):3-7, 2019.

[32] Jorge Rocha and Nair Alves. Osm indoor: moving forward. In OGRS2012-Symposium proceedings, pages 261-167, 2012.

[33] Irene Rubino, Jetmir Xhembulla, Andrea Martina, Andrea Bottino, and G. Malnati. Musa: Using indoor positioning and navigation to enhance cultural experiences in a museum. Sensors (Basel, Switzerland), 13:17445-71, 122013.

[34] Derik Schröter and Michael Beetz. RG Mapping: Building Object-Oriented Representations of Structured Human Environments. 6-th Open Russian-German Workshop on Pattern Recognition and Image Understanding (OGRW), Katun/Russia, pages 1-4, 2004.

[35] Srishti Srivastavaa, Nishith Maheshwarib, and K Rajanc. TOWARDS GENERATING SEMANTICALLY-RICH INDOORGML DATA FROM ARCHITECTURAL PLANS. ISPRS - International Archives of the Photogrammetry, Remote Sensing and Spatial Information Sciences, XLII-4:591-595, September 2018.

[36] Carl Turner, James Lewis, and Jakob Nielsen. Determining Usability Test Sample Size, volume 3. 012006.

[37] Arie van Deursen, Paul Klint, and Joost Visser. Domain-specific languages: an annotated bibliography. ACM SIGPLAN Notices, 35(6):26-36, 2000.

[38] Markus Voelter, Sebastian Benz, Christian Dietrich, Birgit Engelmann, Mats Helander, Lennart C. L. Kats, Eelco Visser, and Guido Wachsmuth. DSL Engineering - Designing, Implementing and Using Domain-Specific Languages. dslbook.org, 2013.

[39] Zhiyong Wang and Lei Niu. A data model for using openstreetmap to integrate indoor and outdoor route planning. Sensors (Switzerland), 18(7):1-19, 2018.

[40] Chenglu Wen, Siyu Pan, Cheng Wang, and Jonathan Li. An Indoor Backpack System for 2-D and 3-D Mapping of Building Interiors. IEEE Geoscience and Remote Sensing Letters, 13(7):992-996, 2016.

[41] Liping Yang and Michael Worboys. Generation of navigation graphs for indoor space. International Journal of Geographical Information Science, 29(10):1737-1756, 2015.

[42] H. Zender, O. Martínez Mozos, P. Jensfelt, G. J.M. Kruijff, and W. Burgard. Conceptual spatial representations for indoor mobile robots. Robotics and Autonomous Systems, 56(6):493-502, 2008. 